Revue européenne des sciences sociales

European Journal of Social Sciences

La sociologie en quête d'une théorie générale

\title{
Introduction : la théorie sociologique générale en question
}

François Chazel et Jacques Coenen-Huther

\section{(2) OpenEdition}

Journals

Édition électronique

URL : http://journals.openedition.org/ress/160

DOI : $10.4000 /$ ress. 160

ISSN : 1663-4446

Éditeur

Librairie Droz

Édition imprimée

Date de publication : 1 février 2008

Pagination : 5-14

ISBN : $978-2-600-01221-8$

ISSN : 0048-8046

Référence électronique

François Chazel et Jacques Coenen-Huther, « Introduction : la théorie sociologique générale en question », Revue européenne des sciences sociales [En ligne], XLVI-140 | 2008, mis en ligne le 01 février 2011, consulté le 19 avril 2019. URL : http://journals.openedition.org/ress/160 ; DOI : 10.4000/ ress. 160

(C) Librairie Droz 


\section{François CHAZEL et Jacques COENEN-HUTHER}

\section{INTRODUCTION: LA THÉORIE SOCIOLOGIQUE GÉNÉRALE EN QUESTION}

Quel que soit le sens qu'on accorde au terme «théorie»- ensemble de propositions à vocation explicative ou cadre de référence à visée structurante - il nous paraît essentiel que subsiste et que se développe en sociologie une activité intellectuelle consistant à élaborer et à perfectionner des théories de portée générale, c'est-à-dire des théories qui prennent pour objet la vie en société dans ses manifestations les plus diverses et non tel ou tel domaine d'activité en particulier. C'est l'avenir de la sociologie comme discipline fondée sur un savoir cumulatif qui en dépend.

De nos jours cependant, la sociologie semble caractérisée toujours davantage par une dispersion intellectuelle et organisationnelle en sociologies thématiques et en domaines de recherche spécialisés. Ceci est fâcheux pour plusieurs raisons. Tout d'abord, la fécondation réciproque des différents champs de recherche s'en trouve compromise. Ensuite, les sociologues de spécialisations diverses tendent à avoir pour partenaires privilégiés, voire exclusifs, les acteurs relevant de leur domaine d'intérêt et non leurs collègues attachés à d'autres objets d'étude, ce qui n'est guère favorable à l'identité disciplinaire de la sociologie. C'est alors la sociologie d'expertise ou sociologie «caméraliste» qui tend à imposer ses priorités basées sur des raisonnements $a d$ hoc. Enfin, dans la mesure où s'affaiblit le pouvoir structurant de la théorie générale, le discours narratif tend fréquemment à se substituer à l'analyse fondée sur un appareil conceptuel préexistant. Et l'on ne compte plus les travaux dont le jargon spécialisé masque mal le caractère strictement descriptif. Tout ceci ne serait pas trop inquiétant si quelques grands paradigmes dominaient clairement la champ de la discipline. Mais la sociologie, en dépit des analogies faciles inspirées d'une lecture superficielle de Thomas Kuhn, est-elle vraiment une discipline pluriparadigmatique? On serait parfois tenté de dire qu'elle en est plutôt à un stade pré-paradigmatique où l'on ne s'entend ni sur son objet ni sur son projet. La persistance de cette situation nous paraît préoccupante et c'est ce qui nous a incité à mettre en chantier le présent volume. Les différentes contributions rassemblées ici abordent directement ou indirectement des aspects fondamentaux de l'élaboration théorique. Elles attirent l'attention - fût-ce de manière implicite - sur des problèmes non encore résolus et des thèmes loin d'être épuisés de la théorie sociologique.

Notre regretté collègue et ami Jean-Michel Berthelot partageait notre inquiétude face au morcellement théorique, épistémologique et conceptuel du champ disciplinaire de la sociologie. Informé de notre projet, sollicité de participer à 
l'entreprise, il avait réagi avec enthousiasme et se promettait de lui apporter une contribution substantielle. Il n'en eut hélas pas le temps. Nous avons donc choisi de publier ici à nouveau sa contribution à un colloque sur le thème «sociologie et relativisme » qui eut lieu à Genève en octobre 2002, en allégeant le texte de propos de circonstances qui auraient été dénués de pertinence dans le présent contexte. Berthelot prend acte du pluralisme apparemment irréductible de la sociologie mais il s'efforce de définir les conditions de la création d'un «espace commun de discussion et d'analyse » porteur d'un espoir de réduction progressive de la diversité des points de vue. Cette réflexion qui acquiert la résonance d'un testament spirituel correspond tout à fait à l'ambition de ce volume auquel nous avons voulu conférer le caractère d'un débat à distance. Autrement dit, on n'a pas cherché ici à faire œuvre de promotion d'école et à rassembler des articles créant une impression d'unanimité factice grâce à la sélection d'auteurs qui seraient tous d'accord sur les fondements et les modalités de l'entreprise sociologique. Les contributions qu'on va lire sont très diverses dans leur visée programmatique comme dans leur mode d'approche de la théorie générale. On s'est efforcé ici de mettre en évidence les questions qu'elles suscitent, en signalant certes les lignes de convergence mais en ne cherchant pas à masquer les divergences par des artifices réthoriques.

Pour enrayer ce qu'il n'hésite pas à qualifier de «cacophonie» des sciences sociales, Jean-Michel Berthelot recommande à la corporation sociologique de se soumettre à une série de contraintes intellectuelles dont on peut espérer qu'elles déboucheront finalement sur un habitus disciplinaire commun. La première de ces contraintes consiste à remettre en évidence la question ontologique. Toute analyse sociologique devrait être présentée de façon à être rendue compatible avec l'idée de «monisme ontologique ». Ceci revient à organiser le passage du particulier au général tout en restant dans un registre conceptuel homogène. Des entités liées à un contexte particulier doivent pouvoir accéder à un niveau de généralité plus large et participer ainsi de la définition même du social. Cette prescription rejoint tout à fait l'ambition de la sociologie formelle telle qu'elle est décrite plus loin par Jacques Coenen-Huther. Elle est également présente, on le verra, dans la stratégie des théories de portée moyenne décrite par Alban Bouvier. Quant à savoir si cette ontologie sociale peut s'articuler fructueusement à une ontologie fondamentale incorporant des mécanismes relevant des sciences de la vie et de la nature, il est peut-être prématuré d'en décider. Mais les avancées de l'éthologie et la progression rapide de la perspective évolutionniste dans la réflexion sur la nature humaine ne permettent certainement plus de tenir la question pour incongrue. La deuxième contrainte à se donner est de s'en tenir à des «modèles d'intelligibilité» relevant d'une causalité sociale, quels que soient les mécanismes en cause. Sans doute, comme le souligne Berthelot, l'explication ne peut ainsi qu'échapper «au simple déterminisme physique ou biologique» mais la maxime traditionnelle «expliquer le social par du social» est inutilement restrictive. L'interpénétration de l'individuel et du social, communément admise actuellement, a pour conséquence que le social émerge du psychologique. Il ne serait pas souhaitable que le monisme ontologique nous ramène au monisme explicatif contre lequel Boudon rompt une lance plus loin. La troisième contrainte à laquelle nous sommes invités à nous soumettre est une exigence de commensurabilité. La pratique de l'argumentation rationnelle exige qu'on se comprenne sans trop de difficultés. La proli- 
fération et la concurrence des terminologies, les innovations de langage qui se donnent pour des innovations conceptuelles, les «postures d'auteur» insuffisamment justifiées font obstacle à la «gestion argumentative» du pluralisme. Ajoutons qu'elles rendent illusoire la construction du savoir cumulatif que nous appelons de nos vœux. La démarche proposée par Berthelot débouche sur l'élaboration de critères de comparaison des théories et des programmes. Trois des contributions rassemblées ici proposent explicitement un programme pour l'élaboration théorique au niveau général: celles de Raymond Boudon, de François Chazel et de Jacques Coenen-Huther. Les trois articles suivants, par Alban Bouvier, Giovanni Busino et Pierre Livet, présentent des points de vue particuliers sur la notion de théorie générale. Enfin, Gérald Bronner et Pierre Demeulenaere choisissent l'angle d'attaque de l'épistémologie et de la méthode.

A l'encontre des conceptions holistes du social qu'il désapprouve, Raymond Boudon esquisse un programme «individualiste» congruent avec son option de méthode. Ce programme vise à traiter les phénomènes sociaux comme les effets de comportements résultant exclusivement de motivations et de raisons. Il est assorti d'une «théorie ouverte de la rationalité » proposant des articulations entre rationalité instrumentale, rationalité cognitive et rationalité axiologique. Dans cette optique, les comportements individuels qui, en dernière analyse, sont à la base de phénomènes complexes, sont des comportements intentionnels et rationnels. La rationalité a ici un caractère axiomatique: l'être humain est un être de raison et l'exercice de la raison porte non seulement sur les moyens mais également sur les fins. Toutefois, dans la mesure où cette conception large du rationnel englobe une relation aux valeurs - la rationalité axiologique -, on peut se demander s'il ne conviendrait pas d'y voir plutôt un postulat de cohérence. Les comportements sont rationnels - ou peuvent tout au moins faire l'objet d'un postulat de rationalité ou de cohérence - mais ils sont également intentionnels : ils sont le fait d'individus échappant au déterminisme de l'instinct et aptes à faire des choix. Lorsque ces choix procèdent de l'adhésion à une valeur, on voit bien comment la délibération rationnelle peut s'exercer dans le cadre d'une échelle de valeurs donnée; on voit moins bien, avouons-le, comment elle peut intervenir dans l'élaboration de cette échelle de valeurs. Faut-il pour autant en conclure que l'acteur est à cet égard le jouet de «forces occultes»? Ce n'est pas ce que suggèrent les travaux de Boudon et cette «bizarrerie» ne peut être entretenue que par des conceptions rudimentaires au point d'en être caricaturales.

Loin d'être des phénomènes opaques se dérobant à l'analyse, les influences émanant de processus de socialisation primaire ou secondaire, d'effets de réseaux, d'attentes de rôles institutionnalisées peuvent, sans s'écarter de la perspective boudonienne, être traduits en termes d'agrégations et d'ajustements de comportements dans le cadre de situations d'interaction. Le programme individualiste souffrirait-il vraiment d'y voir des manifestations d'intersubjectivité pour autant que celles-ci soient clairement définies et analysées? Que les influences subies n'aient pas toujours le même effet - par exemple que les conditions favorables à la délinquance ne produisent pas toujours des délinquants - indique seulement que l'être humain n'est pas la cible passive de forces purement extérieures mais que ses décisions se prennent en interaction avec d'autres humains et sur la base de ces 
interactions. Le programme dont Raymond Boudon se fait le promoteur parait donc confronté à une option qu'on pourrait qualifier de stratégique: s'intéresser prioritairement aux conceptions pouvant être acceptées par tous car elles font l'objet de l'adhésion de chacun ou bien mettre en évidence les processus d'interaction d'où émergent des conceptions partagées. Il n'en reste pas moins que ce programme semble traversé par une tension entre rationalité et intentionnalité. Mais l'attribution de rationalité comporte elle-même des difficultés qui, nous semble-t-il, demandent à être explorées plus avant. Sans doute, comme le note Boudon, les actions, les croyances et les attitudes «peuvent être comprises » mais cette opération mentale de reconstruction de la logique de l'acteur - comme toute opération mentale fondée sur un sentiment d'évidence - présente un risque tout à fait sérieux d'universalisation du local, c'est-à-dire d'élargissement imprudent du domaine de validité d'hypothèses psychologiques communément admises dans un milieu donné et que Gérald Bronner nous invite à considérer d'un regard critique.

Pour François Chazel, le programme de la théorie générale ne peut qu'être centré sur une sociologie de l'action qui considère l'individu comme un agent porteur de rôles et adhérant à des valeurs. En cela, il ne s'écarte pas fondamentalement de la perspective tracée par Boudon mais il accorde davantage d'importance aux conséquences normatives des valeurs et à la dimension normative de l'action, sensible en cela à l'influence parsonienne. La double structuration de l'action - par la dialectique des rôles et attentes de rôles ainsi que par le balisage des valeurs - invite à prendre en compte la perspective théorique fondée sur l'orientation normative de l'action. Ceci n'implique en aucune façon qu'on instaure une dichotomie radicale entre le registre de la rationalité et celui des normes. La référence à la règle - en d'autres termes, la dimension normative de l'action - s'insère de deux façons dans le cadre de référence de l'action rationnelle: d'une part, par la réflexion sur l'acceptabilité de la norme, d'autre part, par les paramètres limitatifs imposés à la relation entre fins et moyens. Le réexamen critique du débat entre Schütz et Parsons, portant sur les orientations fondamentales de la sociologie, est l'occasion pour Chazel de réexplorer cette question essentielle. On convient généralement que la connaissance résulte d'une combinaison entre trois éléments: un sujet, un objet et une structure opératoire. En sciences sociales, c'est la relation entre le sujet et l'objet qui a un caractère problématique; la structure de connaissance y est perméable à la subjectivité quand bien même on s'efforce de neutraliser l'élément subjectif. Schütz et Parsons adoptent à cet égard des attitudes différentes. A la recherche d'un fondement ontologique à la réflexion sociologique, Schütz confère la prééminence au point de vue subjectif qui lui paraît offrir le seul accès adéquat à la réalité sociale. Le monde de la vie quotidienne est pour lui la «réalité dominante» qui s'impose à notre conscience. Dans le cadre de cette réalité dominante, il existe toutefois des domaines de signification distincts, marqués par des modes d'expérience spécifiques. Il peut s'agir notamment du monde de la création artistique ou de la méditation religieuse mais aussi de la réflexion scientifique. Le domaine du raisonnement scientifique est en effet un domaine de signification particulier, caractérisé par un style cognitif et un état de conscience spécifiques. Et c'est, selon Schütz, dans le «domaine de signification» de l'activité scientifique que le concept de 
rationalité trouve sa place. Parsons récuse la dualité entre le point de vue subjectif et le point de vue objectif. L'argumentation rationnelle est sans doute le discours typique du monde de la science mais les attributions de rationalité - quelle qu'en puisse être la fragilité - sont aussi le fait des acteurs engagés dans le monde de la vie quotidienne; d'un domaine de signification à l'autre, ce sont les critères adoptés qui changent. Parsons estime toutefois le fondement ontologique de la sociologie hors d'atteinte. Il s'en tient à une prise de position d'ordre épistémologique et présente, de façon très piagétienne, le cadre de référence de l'action comme un filtre: toute connaissance est partielle, sélective, et met nécessairement en jeu un schème conceptuel. Ce filtrage de la réalité par la structure de connaissance du sujet s'impose quel que soit le domaine de signification envisagé. Le refus de l'opposition subjectif-objectif en est certainement conforté. Mais la précarité de la compréhension sociologique, que Boudon semble sous-estimer, y trouve par là même une confirmation.

Jacques Coenen-Huther propose d'axer la théorie générale sur un programme de sociologie formelle dans la tradition de Simmel, Dupréel et Bouglé. Cette sociologie des formes - qui est en fait une sociologie des formes et des mécanismes - lui paraît offrir la perspective la plus solide de progrès de la théorie sociologique par passage alterné du particulier au général et vice-versa. Il s'agit d'une sociologie relationnelle dont l'unité n'est pas l'individu mais bien la situation d'interaction ou, si l'on préfère, le rapport social. Cette conception relationnelle du social permet de surmonter l'opposition qu'on peut tenir pour surannée entre le holisme et l'individualisme. Une société humaine n'est pas un organisme distinct des individus qui la composent mais elle n'est pas non plus l'addition pure et simple de ces individus. L'imbrication des rapports sociaux provoque des effets émergents qui échappent à la volonté et souvent à la conscience des acteurs. Ces effets émergents sont à la base des structures sociales; on ne peut les réduire à l'agrégation de comportements individuels. La sociologie relationnelle dont Coenen-Huther se fait l'avocat peut être considérée comme une sociologie de l'action mais c'est une sociologie de l'action beaucoup plus sensible aux contraintes structurelles que le programme individualiste proposé par Boudon. Les raisons et les motivations des acteurs y jouent incontestablement un rôle mais ce ne sont pas les seuls facteurs de causalité. Les éléments formels des relations s'imposent aux individus et aux groupes, indépendamment de ce qui les motive, des raisons qu'ils se donnent ou des représentations qu'ils se font de leur action. Les acteurs peuvent faire preuve d'une très grande lucidité ou au contraire se méprendre totalement sur ce qui les anime. Le programme de la sociologie formelle gagnerait en crédibilité théorique si le statut ontologique des formes était clarifié. S'agit-il de contraintes purement extérieures aux individus - l'effet de nombre par exemple - ne mettant en jeu que des effets de système, ou s'agitil au contraire de mécanismes induits par des constantes de l'esprit humain? Les auteurs pouvant être rattachés directement ou indirectement à la tradition du formalisme sociologique se situent tantôt dans le registre de l'analyse systémique, tantôt dans celui de la réflexion sur la nature humaine. Les formes sociales ne constituent pas une catégorie homogène. L'élaboration d'une typologie s'avère indispensable. L'ébauche de classification esquissée par CoenenHuther sur la base de ses propres travaux de terrain constitue un premier pas dans 
cette voie. Mais beaucoup reste à faire pour dépasser l'étape purement programmatique.

Dans une contribution au tour résolument polémique, Alban Bouvier, se référant à la critique mertonienne du modèle parsonien, pose à nouveaux frais la question à laquelle il n'est pas possible de se soustraire: l'élaboration d'une théorie générale est-elle réellement un projet pertinent? Bouvier est très conscient des inconvénients de la fragmentation actuelle du champ disciplinaire de la sociologie mais il n'en souligne pas moins - à juste titre - les défauts graves que comportent des projets théoriques dont l'abstraction et la généralité masquent mal la stérilité. Et l'essentiel de sa démarche consiste à repérer les conditions que doit remplir une théorie générale pour échapper aux inconsistances de la pseudo-généralité. La stratégie mertonienne d'élaboration de middle range theories ou théories de moyenne portée lui paraît à cet égard fructueuse. Encore faut-il se demander comment ces théories - faut-il vraiment les qualifier de modèles? - peuvent se combiner ou s'articuler de façon à constituer une étape intermédiaire et à fournir les bases d'une théorie générale. Ce peut être, par exemple, à la manière de James Coleman, en établissant des liens «horizontaux» entre mécanismes se situant au même niveau d'abstraction ou des liens «verticaux» entre mécanismes hiérarchisables en fonction de leur degré d'abstraction plus ou moins élevé. Il s'agit bien ici d'une réduction par abstraction en vue de s'élever à un niveau plus élevé de généralité. Mais ce processus n'est pas à sens unique: il faut pouvoir descendre au niveau de mécanismes ou de catégories tout à fait élémentaires - ceci fait penser aux unit-acts de Parsons - pour pouvoir s'élever sur cette base réorganisée à un niveau plus général. Bouvier vise à élargir et à préciser l'approche de Coleman sur laquelle il s'appuie. Au cours de cet examen critique, il en vient à la constatation qu'une théorie générale peut s'orienter vers des mécanismes non intentionnels les sentiments - ou vers des mécanismes intentionnels - les motivations fondées sur des raisons. Bouvier explore plus avant la voie intentionnelle. Comme Boudon, il sent la nécessité de l'élargissement de la notion de rationalité mais il propose d'autres solutions que la distinction classique entre rationalité instrumentale et rationalité axiologique. La première serait d'élargir la rationalité instrumentale à d'autres fins que celles qui sont traditionnellement envisagées, par exemple la fidélité à une valeur. Dans ce cas, la notion de rationalité axiologique s'estompe au profit d'une rationalité instrumentale de portée plus large. On peut néanmoins douter que l'analyse y gagne en précision. La seconde solution serait d'apprécier la rationalité sous l'angle de la cohérence dans le temps, par rapport à des choix antérieurs. On retombe ici indirectement sur les problèmes soulevés par l'idée de rationalité limitée dans le cadre des processus de prise de décision. Mais comme on l'a suggéré plus haut, c'est peut-être toute forme de rationalité qui peut faire l'objet d'un jugement de cohérence. Quoi qu'il en soit, une théorie vraiment générale ne peut que surmonter l'alternative entre mécanismes intentionnels et non intentionnels. Une question se pose néanmoins: les deux sources de mécanismes sociaux - sentiments et intentions - sont-elles véritablement exclusives l'une de l'autre? Il est vrai, comme le souligne fort justement Bouvier, que les sentiments naissent de façon non intentionnelle dans une situation d'interaction. Pourtant, ne peuvent-ils pas aussi avoir pour effet de cristalliser des intentions? L'intention de tirer vengeance d'un tort subi ne peut-elle pas surgir de la jalousie 
ou de la colère? La participation à une mobilisation politique ne peut-elle être stimulée par un sentiment d'humiliation? Tout ceci suggère d'ailleurs que la distinction entre mécanismes intrinsèquement sociaux et mécanismes psychologiques reste un objet de discussions tout comme continue à l'être la relation entre la psychologie et la sociologie ainsi qu'en témoignent la plupart des contributions à ce volume. Mais quelle que soit la perspective adoptée, il ne semble pas y avoir d'inconvénient majeur à admettre que le social émerge du psychologique tout comme l'action réfléchie émerge de l'infra-rationnel.

Pour Pareto, tel que nous le présente Giovanni Busino, l'être humain est tout le contraire d'un être de raison et le principe de rationalité ne joue qu'un rôle très limité dans les motivations et les raisons des acteurs, un rôle nettement plus limité que chez Boudon et les boudoniens. Ce sont les sentiments qui façonnent les actions et les comportements. Après coup, ils sont «habillés » de raisonnements qui fournissent des arguments justificatifs. Ceci n'empêche pas que l'on doive pouvoir mettre en évidence des constantes du comportement humain. L'édifice intellectuel de la sociologie paretienne repose en effet, nous rappelle Busino, sur un postulat de stabilité de la nature humaine dont on ne peut imaginer le changement qu'à très long terme, au rythme des processus d'évolution des espèces naturelles. Dès lors, toute activité humaine dépend fondamentalement d'un substrat permanent et ne se présente qu'en surface sous des aspects contingents. C'est la fameuse dichotomie des «résidus » et des «dérivations ». L'existence du substrat, correspondant à des constantes de la nature humaine, combiné à une conception dynamique de l'équilibre social, confère un caractère cyclique aux phénomènes sociaux. C'est ce que Busino s'attache à démontrer en analysant longuement la théorie des élites de Pareto. Au fond, ce qui nous est ironiquement suggéré par cette théorie, c'est que «plus ça change, plus c'est la même chose». La contingence historique empêche cependant d'envisager l'élaboration de véritables lois de la vie en société.

La dualité paretienne de la permanence et du changement présente une certaine parenté avec l'opposition forme-contenu du courant de sociologie formelle sur lequel s'appuie Coenen-Huther. La différence de terminologie peut toutefois susciter la confusion. La perspective paretienne commentée par Busino distingue un «fond» immuable auquel s'opposent des «formes» variables dans la mesure où elles correspondent à des représentations sociales tributaires d'une certaine époque et d'une certaine culture. Dans la tradition formaliste, ce sont les formes - c'est-à-dire les éléments formels des relations sociales - qui constituent l'élément constant, récurrent, alors que le «contenu» des interactions est variable et soumis aux conceptions et croyances du lieu et du moment. Dans les deux cas cependant, on se fonde sur un postulat de stabilité de la nature humaine, au-delà des configurations historiquement changeantes. Dans les deux cas également, l'élément stable des interactions - résidus paretiens ou formes simmeliennes s'insère dans une conception systémique du social, explicite chez Pareto, implicite chez Simmel et ceux qui s'en inspirent.

Dans la vision paretienne, l'élément stable constitue l'aspect latent, caché, de l'activité humaine alors que l'élément variable en est la partie manifeste. Dans 
une certaine mesure, les dérivations, malgré leurs traits contingents, peuvent néanmoins exercer une influence en retour sur la stabilité des résidus. C'est la dialectique du symbolique et du structurel. On se trouve donc face à une relation de dépendance mutuelle. Busino y voit une «matrice» assurant la médiation entre des structures objectives intériorisées et des conduites individuelles. Ceci lui suggère un rapprochement avec la notion d'habitus, telle qu'elle apparaît dans l'œuvre de Pierre Bourdieu. Ce dernier présente en effet l'habitus comme un principe de génération et de structuration des comportements et des représentations. On peut alors y voir un mécanisme de régulation des attitudes et des comportements qui n'implique ni l'obéissance à des règles explicitement formulées ni l'adhésion à une finalité consciemment exprimée. Pour Bourdieu comme pour Pareto, ce qui est latent est fondamental et doit être dévoilé. Au-delà de l'idée d'un facteur caché à mettre en lumière, on peut néanmoins se demander s'il n'y a pas une différence essentielle entre l'élément structurant bourdieusien et la structure latente paretienne. Dans le premier cas, il s'agit d'un «système de dispositions » qui résulte de stratégies de socialisation et d'expériences accumulées; dans le second cas, il est question d'un noyau de tendances dont l'origine réside dans des constantes de la nature humaine. L'hypothèse du substrat immuable semble indispensable aux raisonnements paretiens; elle ne paraît jouer aucun rôle dans la sociologie de Bourdieu.

Pierre Livet s'interroge sur l'apport possible de l'analyse de réseaux à une théorie générale de la vie en société. En d'autres termes, il examine les bénéfices qu'on peut tirer d'une description des relations sociales en termes de réseaux d'interaction. Ce mode d'analyse nous offre une représentation de l'état d'un réseau à un moment donné. Pour l'utiliser au service d'une théorie de la dynamique sociale, Livet a recours à la catégorie du «virtuel», par opposition à l'actuel. Le virtuel, dans ce contexte, se définit à partir de l'actuel. Les événements passés sont virtuels mais ils ont été actuels et leur prise en compte enrichit l'analyse dans la mesure où ils ne sont pas sans influence sur les représentations des individus. On peut se demander toutefois si une distinction stricte s'impose entre les propriétés structurelles des réseaux et la nature des relations qui s'y insèrent. Des groupements sociaux réels - groupes, communautés, institutions, etc - prennent-ils appui sur les éléments formels des réseaux, en sont-ils à l'origine ou au contraire la résultante? Il n'y a probablement pas de réponse simple à cette question. Quand on pratique l'analyse sociologique sur fond de psychologie - il semble vraiment difficile de l'éviter - on se rend compte que les éléments formels des relations contribuent à définir leur nature mais ne peuvent en être détachés que par la pensée. La segmentation des réseaux est inséparable de la ségrégation des rôles sociaux. Un groupe peut se définir formellement comme une configuration de relations mais un double sentiment d'identité et d'altérité émerge inévitablement de cette configuration. Chaque «trou structural» ne crée pas une relation de pouvoir mais une position de pouvoir peut être atteinte grâce à une stratégie impliquant la création ou le maintien de trous structuraux. Les notions durkheimiennes de solidarité mécanique entre individus semblables et de solidarité organique entre individus complémentaires peuvent se traduire par les caractéristiques formelles de réseaux mais elles servent elles-mêmes à définir des relations d'un point de vue strictement formel. La leçon majeure des analyses de Livet pourrait 
bien être que le formel et l'actuel se trouvent dans un état de mutuelle dépendance alimenté par le virtuel.

Gérald Bronner s'interroge sur les catégories mentales qui nous servent à appréhender le réel et n'hésite pas à faire ressurgir la vieille question de la possibilité d'une connaissance objective de la réalité. La relation du sujet connaissant à l'objet de sa connaissance implique toujours une réduction de complexité et une segmentation de la réalité en fonction d'une culture préalable et de centres d'intérêt préexistants. Il n'y a donc pas de connaissance sans présupposés. Les règles de la méthode expérimentale doivent nous guider dans nos imputations causales mais la possibilité de «biais cognitifs » est toujours présente et il est bon de ne pas l'oublier. A ce propos, Bronner, s'inspirant de Karl Popper, souligne les faiblesses de la logique inductive lorsqu'elle s'impose au «jugement de possibilité » weberien mobilisant les ressources de l'expérience au service de l'analyse causale. L'argumentation popperienne mérite certainement l'attention mais on ne peut s'empêcher de penser que les raisonnements sociologiques seraient bien pauvres et la sociologie bien stérile si le raisonnement inductif devait en être banni. C'est Jean-Claude Passeron qui a eu le mérite de nous faire observer que le raisonnement sociologique, en dépit de moments expérimentaux, se développe le plus souvent dans un «espace non popperien». Poursuivant l'examen des processus cognitifs et des biais cognitifs possibles, Gérald Bronner nous invite à considérer la «cartographie de l'erreur» comme un instrument utile au service de la compréhension sociologique, nous rappelant au passage qu'erreur et rationalité ne sont pas antinomiques. Au-delà d'une psychologie de convention, une réflexion approfondie sur nos processus mentaux pourrait constituer un apport non négligeable à l'élaboration théorique.

Dans la même veine épistémologique que Bronner, Pierre Demeulenaere pose le problème de la conceptualisation comme préalable à tout effort théorique. Les notions classiques de «coûts» et «avantages» lui paraissent fournir un socle interprétatif d'application générale pour autant qu'on puisse préciser le contenu de ces notions ainsi que leur degré d'homogénéité pour une catégorie donnée de personnes. Les conceptions théoriques en la matière opposent souvent la poursuite d'intérêts personnels au respect de normes sociales ainsi qu'à la soumission à des obligations collectives. On peut se demander toutefois - et c'est la question que soulève Demeulenaere - si un tel contraste conserve une réelle valeur analytique. L'interpénétration de l'individuel et du social porterait plutôt à distinguer différentes modalités et différents degrés de pression sociale, les préférences dites «individuelles» relevant de normes intériorisées de longue date et d'influences diffuses alors que d'autres normes, dites «sociales», font l'objet de pressions normatives plus nettes, allant du poids de l'opinion jusqu'aux règles juridiques assorties de sanctions. Quoi qu'il en soit, il y a lieu de s'interroger à nouveau sur la notion d'utilité et sur l'indétermination qui s'y associe. Différents scénarios relatifs aux préférences - préférences hétérogènes, préférences identiques ou préférences homogènes mais d'intensité variable - peuvent être traduits en termes de coûts et avantages. Ceci apparaît tout particulièrement dans la relation d'échange qui oppose deux «volontés maximisatrices». Toutefois, c'est ici que des considérations normatives exercent une effet de régulation. Toute préférence 
a un aspect évaluatif et, en ce sens, normatif. Et c'est ainsi que le clivage entre l'individuel et le collectif perd de son acuité.

A l'examen des travaux en chantier que nous présentons ici, quelques questions nous paraissent primordiales pour l'avenir de la théorie générale. Tout d'abord, en amont de l'élaboration théorique, une réflexion sur la nature des invariants s'impose plus que jamais. Certains doutent qu'il faille s'en tenir à cet égard à un postulat de stabilité. Il nous semble plutôt y voir une condition sine qua non de tout effort théorique sérieux. Ensuite, ne convient-il pas de promouvoir une conception relationnelle de la sociologie, incluant l'analyse de manifestations d'intersubjectivité? Une telle orientation nous paraît éminemment fructueuse pour l'étude de processus d'interaction d'où émergent finalement des conceptions partagées. Enfin, la compréhension sociologique, en tant qu'effort de reconstruction de logiques d'action, ne devrait-elle pas faire l'objet d'un réexamen approfondi? L'enjeu reste évidemment la question désormais classique du rapport du sujet connaissant à l'objet de sa connaissance. Mais c'est une question qui n'a pas encore reçu de réponse rassurante du côté de la sociologie. 